Research Paper

\title{
Permethrin Induction of Multiple Cytochrome P450 Genes in Insecticide Resistant Mosquitoes, Culex quin- quefasciatus
}

\author{
Youhui Gong ${ }^{1,2}$, Ting $\mathrm{Li}^{1}$, Lee Zhang ${ }^{1,3}$, Xiwu Gao ${ }^{2 \bowtie}$ and Nannan Liu ${ }^{1 \bowtie}$ \\ 1. Department of Entomology and Plant Pathology, Auburn University, Auburn, AL 36849, USA. \\ 2. Department of Entomology, China Agricultural University, Beijing, China. \\ 3. Genomics and Sequencing Laboratory, Auburn University, Auburn, AL 36849, USA.
}

$\triangle$ Corresponding author: Dr. Nannan Liu, Department of Entomology and Plant Pathology, Auburn University, Auburn, AL 36849, USA. liunann@auburn.edu or Dr. Xiwu Gao, Department of Entomology, China Agricultural University, Beijing, China.

( ) Ivyspring International Publisher. This is an open-access article distributed under the terms of the Creative Commons License (http://creativecommons.org/ licenses/by-nc-nd/3.0/). Reproduction is permitted for personal, noncommercial use, provided that the article is in whole, unmodified, and properly cited.

Received: 2013.05.22; Accepted: 2013.08.17; Published: 2013.09.05

\begin{abstract}
The expression of some insect P450 genes can be induced by both exogenous and endogenous compounds and there is evidence to suggest that multiple constitutively overexpressed P450 genes are co-responsible for the development of resistance to permethrin in resistant mosquitoes. This study characterized the permethrin induction profiles of P450 genes known to be constitutively overexpressed in resistant mosquitoes, Culex quinquefasciatus. The gene expression in 7 of the 19 P450 genes CYP325K3vl, CYP4D42v2, CYP9]45, (CYP) CPIJ000926, CYP325G4, CYP4C38, CYP4H40 in the HAmCqG8 strain, increased more than 2-fold after exposure to permethrin at an LC50 concentration (10 ppm) compared to their acetone treated counterpart; no significant differences in the expression of these P450 genes in susceptible S-Lab mosquitoes were observed after permethrin treatment. Eleven of the fourteen P450 genes overexpressed in the MAmCqG6 strain, CYP9MI0, CYP6ZI2, CYP9J33, CYP9J43, CYP9]34, CYP306AI, CYP6ZI5, CYP9j45, CYPPALI, CYP4C52vI, CYP9]39, were also induced more than doubled after exposure to an LC50 (0.7 ppm) dose of permethrin. No significant induction in P450 gene expression was observed in the susceptible S-Lab mosquitoes after permethrin treatment except for CYP6ZI5 and CYP9]39, suggesting that permethrin induction of these two P450 genes are common to both susceptible and resistant mosquitoes while the induction of the others are specific to insecticide resistant mosquitoes. These results demonstrate that multiple P450 genes are co-up-regulated in insecticide resistant mosquitoes through both constitutive overexpression and induction mechanisms, providing additional support for their involvement in the detoxification of insecticides and the development of insecticide resistance.
\end{abstract}

Key words: Permethrin induction, P450 genes, detoxification, insecticide resistance, Culex quinquefasciatus.

\section{Introduction}

Cytochrome P450s have long been of particular interest because of their critical role in the detoxification and/or activation of xenobiotics such as drugs, pesticides, plant toxins, chemical carcinogens and mutagens; and in metabolizing endogenous com- pounds such as hormones, fatty acids, and steroids [1, 2]. In many cases, the overexpressed $P 450$ proteins and P450 activity that result from constitutive transcriptional overexpression of $\mathrm{P} 450$ genes in insecticide resistant insects have been shown to result in the en- 
hanced metabolic detoxification of insecticides in insects [3, 4]. Overexpression of P450 genes has been linked to the development of resistance in many insect species, including Musca domestica [5-10], Culex quinquefasciatus [11-13], Culex pipiens quinquefasciatus [14], Drosophila [15, 16], Aedes aegypti [17], Anopheles gambiae [18], Helicoverpa armigera [19], Tribolium castaneum [20], Bemisia tabaci [21, 22], and Trialeurodes vaporariorum [23]. In a recent study, Reid et al. [24] used RNAseq methodology to conduct a whole transcriptome analysis of Culex quinquefasciatus in permethrin resistant mosquitoes and found that the overrepresented functional categories were mainly among the P450 genes, which may indicate that the overexpression of multiple P450 genes is a key factor governing the increased levels of detoxification of insecticides and insecticide resistance in Culex mosquitoes.

Another interesting feature of some P450 genes is that their expression can be induced by both exogenous and endogenous compounds, a phenomenon known as induction. This is a major concern due to the enhanced metabolism of pharmaceutical drugs, insecticides and endogenous compounds [3, 4, 25]. Many studies have suggested that the phenomenon of induction of P450s in insects plays a critical role in the metabolism of plant toxins and may also facilitate the adaptation of insects to their host-plants [26-32]. There is also evidence to suggest that the P450 induction mechanism is involved in the detoxification of insecticides and xenobiotics in insects species such as Diabrotica virgifera virgifera [33], Drosophila melanogaster [34], Musca domestica [6, 35], Plutella xylostella [36], Aedes aegypti [37, 38] and Culex quinquefasciatus [13]. Induction of one or more P450 genes has been proposed as a potential factor that results in insecticide resistance in these insects and both constitutively increased expression and induction of P450s are thought to be responsible for increased levels of detoxification of insecticides [33, 39, 40]. Moreover, variations in the induction levels of P450 genes are likely to exist between different species, development stages, and insecticide susceptible or resistant strains, and are thought to be responsible, in part at least, for the selective toxicity of insecticides, the development of resistance to insecticides, and the selection of host plants by insects [39]. The mosquito Culex quinquefasciatus is a primary vector of West Nile encephalitis, eastern equine encephalitis, Saint Louis encephalitis, and lymphatic filariasis pathogens [41-44]. Pyrethroids such as permethrin are currently the most widely used insecticides for the indoor control of mosquitoes worldwide and the only chemical recommended for the treatment of mosquito nets, the main tool for preventing malaria in Africa [45]. Nevertheless, the development of resistance to insecti- cides, especially to pyrethroids, in mosquito vectors has become a global problem [46-51]. Our previous studies on whole transcriptome analyses of insecticide resistance [24] and genome analyses of cytochrome P450s and their expression profiles [12] in insecticide resistant mosquitoes have identified multiple up-regulated P450 genes in Cx. quinquefasciatus that may be responsible for the development of resistance to insecticides such as permethrin. In the current study, the induction profiles of multiple up-regulated P450 genes identified in highly resistant mosquitoes HAmCqG8 and MAmCqG6 have been examined to further test the hypothesis that both induction and constitutive overexpression of P450s are involved in insecticide resistance by increasing the overall expression levels of P450 genes.

\section{Materials and methods}

\section{Mosquito strains}

Three strains of Culex quinquefasciatus were used in this study. The insecticide susceptible S-Lab strain was obtained from Dr. Laura Harrington (Cornell University) and used as a reference strain for comparing the resistance levels to permethrin among the different strains. $\mathrm{HAmCq}{ }^{\mathrm{G}}$, the $8^{\text {th }}$ generation of permethrin-selected offspring of $\mathrm{HAmCq}{ }^{\mathrm{G} 0}$, a field resistant strain collected from Madison County, Alabama, USA [52], has exhibited a 2700-fold level of resistance [53] and $\mathrm{MAmCqG6}$, the $6^{\text {th }}$ generation of permethrin-selected offspring of $\mathrm{MAmCq}^{\mathrm{G} 0}$, a field resistant strain collected from Mobile County, Alabama, USA [52], has a 570-fold level of resistance compared with the susceptible S-Lab strain [53]. $\mathrm{HAmCqG0}$ and MAmGqG0 were collected from sites located $>600 \mathrm{~km}$ apart in the state of Alabama, USA. All the mosquitoes were reared at $25 \pm 2^{\circ} \mathrm{C}$ under a photoperiod of 12:12 (L:D) h [54], and fed blood samples from horses (Large Animal Teaching Hospital, College of Veterinary Medicine, Auburn University).

\section{Permethrin Treatment}

Our previous study revealed a clear concentration dependent response of P450 genes to permethrin treatment in Culex mosquitoes, with maximum levels of induction of P450 genes at a permethrin concentration of $\mathrm{LC}_{50}$ [13]. Accordingly, in the current study the induction of P450 genes in response to permethrin was determined by treating 1000 late $3^{\text {rd }}$ instar larvae of each of the three Culex mosquito strains with permethrin at their respective $\mathrm{LC}_{50}$ concentrations $(0.005$ ppm, 0.7 ppm, and 10 ppm for the S-Lab, MAmCqG6, and $\mathrm{HAmCq}{ }^{\mathrm{G} 8}$ strains, respectively). The surviving mosquitoes were collected for RNA extraction 12, 24, 
48 and $72 \mathrm{~h}$ after permethrin challenge and their expression of the P450 genes was examined. Control mosquitoes exposed to $1 \%$ acetone were collected at the same time points as their permethrin treatment counterparts. The experiments were repeated three times.

\section{RNA extraction and cDNA preparation}

The total RNA was extracted from the surviving permethrin treated and acetone treated (control) mosquitoes using the acidic guanidine thiocyanatephenol-chloroform method [6]. The DNA was removed from the total RNA $(5 \mu \mathrm{g})$ of each mosquito sample using DNase (TURBO DNA-free, Ambion) and the DNA-free total RNA (0.5 mg per sample) reverse-transcribed to cDNA using Transcriptor First Strand cDNA Synthesis kit (Roche) and a random hexamer primer following the manufacturer's instructions. The quantity of cDNA was measured by a spectrophotometer prior to qRT-PCR. Each experiment was repeated three times with RNA preparation and cDNA synthesis.

\section{Quantitative Real-time PCR (qRT-PCR)}

The induction of $\mathrm{P} 450$ gene expression was performed with the SYBR Green master mix Kit and ABI 7500 Real Time PCR system (Applied Biosystems). In this study, we tested 19 and 14 P450 genes that were over-expressed in the larvae of resistant HAmCqG8 and MAmCqG6, respectively, as reported by Yang and Liu [12]. Among these P450 genes, 4 P450 genes (CYP6P14, CYP4C52v1, CYP9AL1, and CYP9J45) were overexpressed in both $\mathrm{HAmCq}^{\mathrm{G} 8}$ and MAmCqG6 strains (Yang, et al. 2011) and tested for their induction in both $\mathrm{HAmCq}^{\mathrm{G} 8}$ and $\mathrm{MAmCq}^{\mathrm{G} 6}$ strains. Other two P450 genes, CYP9J34 and CYP9M10 that were also overexpressed in $\mathrm{HAmCq} 8$ and $\mathrm{MAmCq}^{\mathrm{G} 6}$ strains, were only tested in in MAmCqG6 because the induction of these two gens had been tested in by Liu, et al. [13] in HAmCqG8. Each qRT-PCR reaction $(25 \mu \mathrm{L}$ final volume) contained $1 \times$ SYBR Green master mix, $1 \mu \mathrm{L}$ of cDNA, and a P450 gene specific primer pair (Table 1) designed according to each of the P450 gene sequences as described previously [12] at a final concentration of 3-5 $\mu \mathrm{M}$. All samples, including the A 'no-template' negative control, were performed in triplicate. The reaction cycle consisted of a melting step of $50^{\circ} \mathrm{C}$ for $2 \mathrm{~min}$ then $95^{\circ} \mathrm{C}$ for $10 \mathrm{~min}$, followed by 40 cycles of $95^{\circ} \mathrm{C}$ for $15 \mathrm{sec}$ and $60^{\circ} \mathrm{C}$ for $1 \mathrm{~min}$. Specificity of the PCR reactions was assessed by a melting curve analysis for each PCR reaction using Dissociation Curves software [55]. Relative expression levels for the P450 genes were calculated by the 2- $\Delta \Delta \mathrm{CT}$ method using SDS RQ software [56]. The $18 \mathrm{~S}$ ribosome RNA gene, an endogenous control, was used to normalize the expression of target genes [9, 57]. Preliminary qRT-PCR experiments revealed that the $18 \mathrm{~S}$ ribosome RNA gene expression remained constant among all 3 mosquito strains, so the $18 \mathrm{~S}$ ribosome RNA gene was used for internal normalization in the qRT-PCR assays. Each experiment was repeated three times with different preparations of RNA samples. The statistical significance of the gene expressions was calculated using a Student's $t$-test for all 2-sample comparisons and a one-way analysis of variance (ANOVA) for multiple sample comparisons (SAS v9.1 software); a value of $P \leq 0.05$ was considered statistically significant. Significant overexpression was determined using a cut-off value of a $\geq 2$-fold change in expression [58].

\section{Results}

\section{Expression of $\mathrm{P} 450$ genes in response to per- methrin challenge in resistant $\mathrm{HAmCq}^{\mathrm{G8}}$ mosquitoes}

Nineteen P450 genes that were over-expressed in the larvae of resistant $\mathrm{HAmCq}^{\mathrm{G} 8}$ strain [12] were tested in this study. The results of an earlier study revealed that although no significant induction was detected in the susceptible S-Lab mosquitoes for the doses $\left(\mathrm{LC}_{10}, \mathrm{LC}_{50}\right.$, and $\mathrm{LC}_{90}$ for each strain) and time intervals $(12,24,48$, and $72 \mathrm{~h})$ tested, permethrin induced three P450 genes CYP6AA7, CYP9J34, and CYP9M10 in resistant HAmCqG8 mosquitoes, reaching a maximum at a permethrin concentration of $\mathrm{LC}_{50}$ [13]. Based on these data, a permethrin concentration of $\mathrm{LC}_{50}$ for each mosquito strain was chosen for the current induction study and the expression of nineteen P450 genes in response to permethrin challenge in each of mosquito strains was characterized for various durations/time intervals (12, 24, 48, and 72h, see Materials and Methods). Examining the durations of P450 gene induction revealed no significant induction in the expression of all these P450 genes in the susceptible S-Lab mosquitoes that had been treated with either acetone alone (control) or with permethrin $\mathrm{LC}_{50}$ concentration $(0.005 \mathrm{ppm})$ at any time points after the treatment (Fig. 1). In contrast, the results reported here revealed that seven of the $19 \mathrm{P} 450$ genes that were up-regulated in HAmCqG8, CYP325K3v1, CYP4D42v2, CYP9J45, (CYP) CPIJ000926, CYP325G4, CYP4C38, CYP4H40, had their gene expression significantly induced by permethrin at an $\mathrm{LC}_{50}$ concentration (10 ppm; $\left.{ }^{*} p \leq 0.05\right)$, climbing to more than double that observed into their acetone treated counterpart, i.e., control mosquitoes (Fig. 1A-G). Interestingly, the expression of these seven genes was induced ( $\geq 2$-fold) at varying levels for different times ranging from 12 to $72 \mathrm{~h}$ in permethrin treated $\mathrm{HAmCq}^{\mathrm{G} 8}$. The 
initial induction for these P450 genes was observed after $12 \mathrm{~h}$ permethrin treatment except for CYP325G4 and CYP9J45, where they occurred after $24 \mathrm{~h}$ permethrin treatment (Figs.1C, 1E). Except CYP4H40, where the maximum expression (3.3-fold induction) was reached $72 \mathrm{~h}$ after exposure (Fig. 1G), the remaining six P450 genes, CYP325K3v1 (Table 1, Fig. 1A), CYP4D42v2 (Fig. 1B), CYP9J45 (Fig. 1C), CYP325G4 (Fig. 1E), CYP4C38 (Fig. 1F), and the P450 gene, CPIJ000926 (un-named, Fig. 1D) peaked at induction levels of 3.9-, 8.8-, 2.5-, 3.1-, 2.1- and 2.9-fold, respectively, $48 \mathrm{~h}$ after permethrin treatment. When measured $72 \mathrm{~h}$ after treatment, the induction levels of CYP325K3v1, CYP4D42v2, CYP9J45, CYP325G4, and CYP4C38 had declined to the point where no significant induction $\left({ }^{*} p \leq 0.05\right)$ compared with acetone treated mosquitoes could be detected (Figs. 1A, 1B, $1 \mathrm{C}, 1 \mathrm{E}, 1 \mathrm{~F})$. In contrast, at $72 \mathrm{~h}$ after treatment CPIJ000926 and CYP4H40 retained their maximum induced levels (Figs. 1D and 1G).

Table I. Up-regulated P450 genes and oligonucleotide primers used in qRT-PCR reactions for P450 gene expression/induction analysis.

\begin{tabular}{|c|c|c|c|c|c|c|}
\hline strain & $\begin{array}{l}\text { Transcript } \\
\text { ID }^{a}\end{array}$ & Accession No. & gene $^{b}$ & $\begin{array}{l}\text { up-reg } \\
\text { ulation } \\
\text { ratio }^{c}\end{array}$ & Forward Primer (5' t & Reverse Primer ( $5^{\prime}$ to $\left.3^{\prime}\right)$ \\
\hline \multirow{19}{*}{$\begin{array}{l}\mathrm{HAm} \\
\text { CqG8 }\end{array}$} & CPIJ017021 & XM_001867284.1 & CYP325K3v1 & 11 & AAGGTCAAGTTTGCCGAGTCGTTG & AAATCTCGTCCGAACTCTTCCCGA \\
\hline & СРIJ020229 & XM_001870408.1 & CYP4D42v2 & 7.0 & GAGCTTCAAATTTGGCCCGCCATT & ACTTCCGGCTGAGGTTCGTTATGA \\
\hline & CPIJ001759 & XM_001843572.1 & CYP4H40 & 7.0 & TTTCCTGTATCCTTTCGCACGGGA & TGTAAACGTCCTCTTCCTGCTGCT \\
\hline & CPIJ005955 & XM_001847351.1 & CYP6P14 & 6.3 & AAGGTGGAACCAGGTCTGACGATT & CCATCATTAGCCGCGATTGCCTTT \\
\hline & CPIJ018943 & XM_001869165.1 & CYP4C52v1 & 6.2 & CACGCCGGCATTCCACTTTAAGAT & GGATTCCCATGGCGGTTTCACAAA \\
\hline & CPIJ010227 & XM_001855535.1 & CYP12F13 & 5.5 & TGGACAAGAATCCGAGCCAAGACA & TCTCCGGGTTCTTTGCTAAGCAGT \\
\hline & CPIJ005954 & XM_001847350.1 & CYP6CC2 & 4.2 & ACGTGTTCTTCTCGCCATCGATCA & AGCTTATCCCTGTACAAACCGCGA \\
\hline & CPIJ012470 & XM_001862711.1 & CYP9AL1 & 3.8 & TGAACGTCCTTAGGGATGGCGAAA & TTGCTAGTCGCGGAAACGAACTGA \\
\hline & CPIJ010537 & XM_001855163.1 & СҮР9J45 & 3.8 & TCAGCGGTACGGAAACGATGTGAT & AGTCCATGTTGGTCTTCTGTCCCA \\
\hline & CPIJ005956 & XM_001847352.1 & CYP6BZ2 & 3.7 & ACCATGGCGTCAGCTAAGGATGAA & TCCGTGGTGAATCCGACTAGCAAA \\
\hline & CPIJ010540 & XM_001855175.1 & СУР9J35 & 3.5 & TTGCCCAATGCTTCCTGTTCTTCC & GTTTCCGCCAAGTGCTTGTTCTGT \\
\hline & CPIJ009478 & XM_001851374.1 & CYP4D42v1 & 3.2 & TCAACTATCTGGTTCGGGATGCGA & ACTTCCGGCTGAGGTTCGTTATGA \\
\hline & CPIJ016847 & XM_001867237.1 & CYP6CQ1 & 3.1 & GCGCTGAAACAGAATCGCGATCAA & TGTCCTTCCTTGGATTCGTTGGGA \\
\hline & CPIJ000926 & XM_001842497.1 & - & 3.1 & GCAAGCAGTACAAATTCGCCGACA & TGATCAGATCGGTCCGTTGCAAGT \\
\hline & CPIJ005957 & XM_001847353.1 & CYP6AA9 & 2.8 & TCACCTGTGACGTCATTGGATCGT & AGGAAGCTGTCGAAGACCCAACTT \\
\hline & CPIJ015957 & XM_001866311.1 & CYP325G4 & 2.8 & CGAGGTGCACGCTGGATTTGATTT & AGTCTTGTGGTAAACCCAGTCCGT \\
\hline & CPIJ001810 & XM_001843432.1 & CYP4C38 & 2.4 & ACTTCAACATCCTGGACGGGTTCT & TCATGGCCGTCTCGCAGATAATGT \\
\hline & CPIJ003082 & XM_001844416.1 & СҮР9J42 & 2.2 & TGCAAGCTAGAGCTGGTCTGCTAA & TGCTTGTGCGATCATTTCCGTGTC \\
\hline & CPIJ002538 & XM_001844155.1 & CYP6AG12 & 2.1 & AAGCCCTTTCAACGCTGGTTGATG & GAGCGTCGATGGCAAACACACAAT \\
\hline \multirow{14}{*}{$\begin{array}{l}\text { MAm } \\
\text { CqG6 }^{\mathrm{G}}\end{array}$} & CPIJ014218 & XM_001864262.1 & CYP9M10 & 1.6 & TGCAGACCAAGTGCTTCCTGTACT & AACCCACTCAACGTATCCAGCGAA \\
\hline & CPIJ004411 & XM_001845945.1 & CYP6Z12 & 2.9 & GCCGGCAAGAAGCTGGAACATTAT & TCCGGGTTGTTCAACGTATCCACT \\
\hline & CPIJ010544 & XM_001855191.1 & СУР9Ј33 & 4.8 & TCCGGCCTTTACTGGTTCCAAGAT & AAAGGCACACGTCGCAATCACATC \\
\hline & CPIJ005332 & XM_001846722.1 & СҮР9J43 & 2.8 & TGTGCCTTCGGTGTTAGACTGGAT & TCGATTCCAAGTTTCCCGGTGAGT \\
\hline & CPIJ010546 & XM_001855208.1 & СYР9J34 & 3.1 & ATCCGATGTCGGTAAAGTGCAGGT & TGTACCTCTGGGTTGATGGCAAGT \\
\hline & CPIJ005958 & XM_001847354.1 & CYP6AA8 & 2.6 & AAAGGCGTTGGCGAAGAGATTCAC & TCGTCAGCAGTTCCGAACCTTCAT \\
\hline & CPIJ001039 & XM_001842656.1 & CYP306A1 & 2.6 & GTGTTTGCCTTTGATCCTGCACCA & GGCAGGAAATTGACCGCCATTGAA \\
\hline & CPIJ005959 & XM_001847355.1 & CYP6AA7 & 2.7 & ATGACGCTGATTCCCGAGACTGTT & TTCATGGTCAAGGTCTCACCCGAA \\
\hline & CPIJ005955 & XM_001847351.1 & CYP6P14 & 2.7 & AAGGTGGAACCAGGTCTGACGATT & CCATCATTAGCCGCGATTGCCTTT \\
\hline & CPIJ008566 & XM_001850110.1 & CYP6Z15 & 3.0 & TCTCGAACGTTCCGGAGGTCAAAT & ATAGTCCTGGGTGCACTTTCGGTT \\
\hline & CPIJ010537 & XM_001855163.1 & СҮР9J45 & 3.1 & TCAGCGGTACGGAAACGATGTGAT & AGTCCATGTTGGTCTTCTGTCCCA \\
\hline & CPIJ012470 & XM_001862711.1 & CYP9AL1 & 3.8 & TGAACGTCCTTAGGGATGGCGAAA & TTGCTAGTCGCGGAAACGAACTGA \\
\hline & CPIJ018943 & XM_001869165.1 & CYP4C52v1 & 14 & CACGCCGGCATTCCACTTTAAGAT & GGATTCCCATGGCGGTTTCACAAA \\
\hline & CPIJ010548 & XM_001855214.1 & CYP9J39 & 2.2 & AGGTACTTGTGTGTGGTTTCCGGT & TGTTTGTTCTCATCGCTGAACCGC \\
\hline
\end{tabular}

a The transcript ID number from the vectorbase of the Cx. quinquefasciatus genome sequence [http://cquinquefasciatus.vectorbase.org/] (Yang and Liu, 2011). $\mathrm{b}$ The annotation of the Culex P450 genes from http://drnelson.utmem.edu/CytochromeP450.html (Yang and Liu, 2011). ${ }^{\mathrm{c}}$ The ratio of the relative gene expression in permethrin selected strain HAmCq ${ }^{\mathrm{GB}}$ compared with its parental strain $\mathrm{HAmCq} \mathrm{G}^{\mathrm{G}}$, data cited from Yang and Liu (2011). 

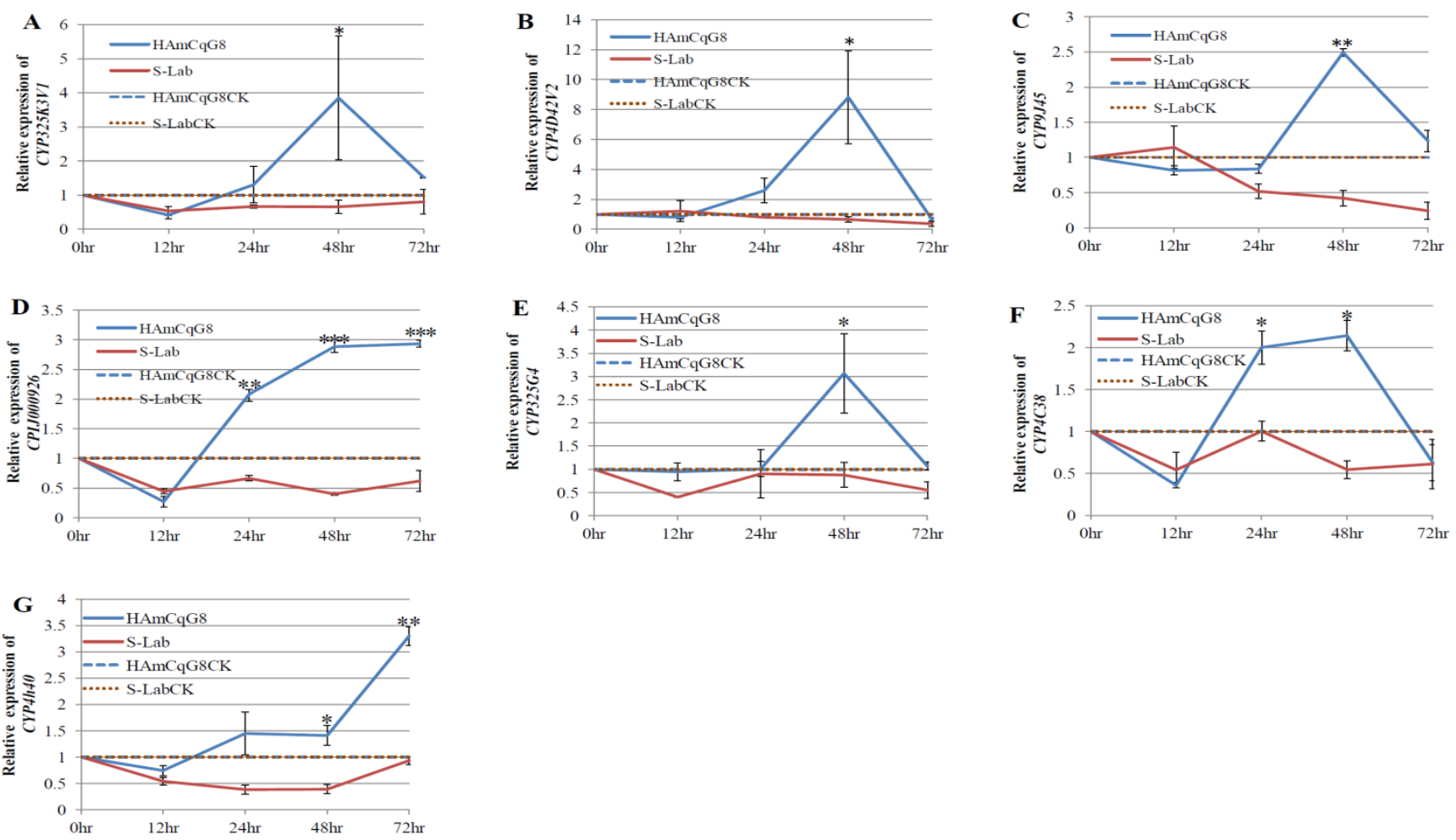

Fig I. The induction of $\mathrm{P} 450$ gene expression in $\mathrm{HAmCq}^{\mathrm{G} 8}$ and S-Lab strains of $C x$. quinquefasciatus following permethrin treatment. The relative expression of P450 genes in Culex mosquitoes following treatment with permethrin at their respective LC $_{50}$ concentrations $(0.005 \mathrm{ppm}$ and $10 \mathrm{ppm}$ for the S-Lab and $\mathrm{HAmCq}{ }^{G 8}$ strains, respectively) were analyzed $12,24,48$, and $72 \mathrm{~h}$ after the permethrin treatment. The relative levels of gene expression shown along the $Y$ axis represent the ratio of the gene expression in each treatment in comparison with that in acetone treated control mosquitoes (noting that the $Y$ axis vary among the sub-figures). The experiments were repeated three times. The results are shown as the mean $\pm \mathrm{S}$.E. The significant difference was indicated by $*(P \leq 0.05), * *(P \leq 0.0 I)$, and $* * *(P \leq 0.00 I)$. A. CYP325K3vI, B. CYP4D42v2, C. CYP9J45, D. (CYP) CPIJ000926, E. CYP325G4, F. CYP4C38, G. CYP4H40.

\section{Expression of $\mathbf{P} 450$ genes in response to per- methrin challenge in resistant $\mathrm{MAmCq}^{\mathrm{G} 6}$ mosquitoes}

We examined the induction of a total of 14 up-regulated $\mathrm{P} 450$ genes in another resistant mosquito population, MAmCqG6 (Table 1) [12] following the same method as that utilized for HAmCqG8. A permethrin concentration of $\mathrm{LC}_{50}(0.7 \mathrm{ppm})$ was used to treat $\mathrm{MAmCq}^{\mathrm{G} 6}$ mosquitoes and the expression of fourteen P450 genes in response to permethrin challenge was characterized at 12, 24, 48, and $72 \mathrm{~h}$ after permethrin treatment. Eleven of the 14 P450 genes known to be up-regulated in MAmCqG6 [12], CYP9M10, CYP6Z12, CYP9J33, CYP9J43, CYP9J34, CYP306A1, CYP6Z15, CYP9J45, CYPPAL1, CYP4C52v1, and CYP9J39, had their gene expression further induced by permethrin at an $\mathrm{LC}_{50}$ concentration $\left(0.7 \mathrm{ppm} ;{ }^{*} p \leq 0.05\right)$ to more than 2 -fold by permethrin compared to the acetone treated control mosquitoes (Fig. 2A-K). Once again, the expression of these eleven genes was observed to vary for times from12 to $72 \mathrm{~h}$ after permethrin treatment in MAmCqG6. Except CYP4C52v1, whose maximum induced expression (2.2-fold induction) reached at $12 \mathrm{~h}$ (Fig 2J), CYP9J43, which had two induced maxima
(2.7- and 2.7-fold induction) at 24 and $48 \mathrm{~h}$ (Fig. 2D), the induction for the remaining nine $\mathrm{P} 450$ genes reached their maxima at $24 \mathrm{~h}$ and $72 \mathrm{~h}$ after permethin treatment. These results are a little different from those in HAmCqG8 mosquitos, where the induction of most of up-regulated P450s by permethrin peaked at $24 \mathrm{~h}$ after treatment. Significant induction levels of CYP9M10, CYP9J33, CYP9J45, and CYP9J39 (Figs. 2A, $2 \mathrm{C}, 2 \mathrm{H}, 2 \mathrm{~K}$ ) in the MAmCqG6 strain were found $24 \mathrm{~h}$ after permethrin treatment, with maximum induction levels of 2.5-, 4.1-, 2.7- and 2.0-fold, respectively. The expression of CYP6Z12, CYP9J34, CYP306A1, CYP9AL1 (Figs. 2B, 2E, 2F, 2I) reached 2.6-, 2.5-, 4.8-, and 3.4-fold levels of induction, respectively, $72 \mathrm{~h}$ after permethrin treatment in MAmCqG6.

In contrast to the induction of $\mathrm{P} 450$ genes in HAmCqG8 mosquitoes, where there was no significant induction in the expression of P450 genes in the susceptible S-Lab mosquitoes treated with permethrin, several of the $\mathrm{P} 450$ genes identified in MAmCqG6 also showed some level of induction in S-Lab mosquitoes treated with permethrin at certain time points. A 1.5-fold lower level of induction of CYP6Z12 was identified $12 \mathrm{~h}$ after permethrin treatment in S-Lab mosquitoes (Fig. 2B), with no corresponding induction in permethrin treated MAmCqG6 However, the 
MAmCqG6did show significant induction of CYP6Z12 from 24 to $72 \mathrm{~h}$ after permethrin treatment and no induction was found in S-Lab at these time points. Low levels of induction of CYP9J33 and CYP9J34 were also detected in the susceptible S-Lab strain after $48 \mathrm{~h}$ permethrin treatment, though statistical significance was not achieved compared to the corresponding levels of induction in the resistant MAmCqG6 strain (Figs. 2C and 2E). Although induction of CYP9J43 was detected in the susceptible S-Lab strain at 12, 24, and $48 \mathrm{~h}$ time points after permethrin treatment, at levels 1.5-, 1.6- and 2.0-fold, respectively (Fig. 2D), the in- duction of CYP9J43 was more evident in the MAmCqG6 strain with levels of 2.7-, 2.7-, and 2.1-fold at 24,48 and $72 \mathrm{~h}$, respectively, after permethrin treatment. The expression of CYP6Z15 (Fig. 2G) and CYP9J39 (Fig. 2K) were both induced significantly in the susceptible S-Lab strain, with induction levels of 3.5 -fold at $48 \mathrm{~h}$ and 4.9 -fold at $72 \mathrm{~h}$, respectively, after permethrin treatment. These results may suggest that some P450 genes are common to the response of both susceptible and resistant mosquitoes to permethrin whereas others are more specific, presenting only in resistant mosquitoes in response to insecticides.
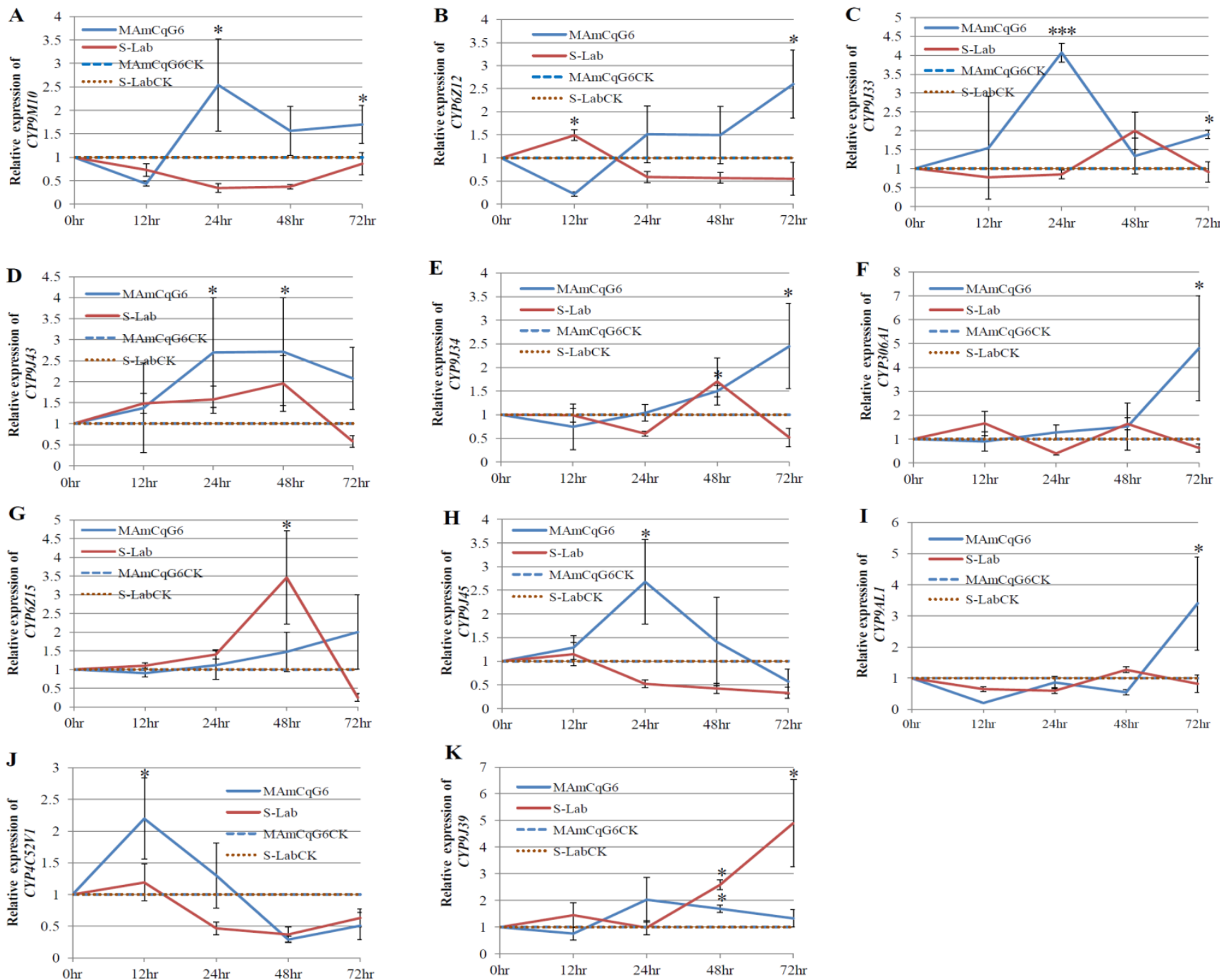

Fig 2. The induction of $\mathrm{P} 450$ gene expression in $M A m \mathrm{Cq}^{\mathrm{G}}$ and S-Lab strains of $C x$. quinquefasciatus following permethrin treatment. The relative expression of $\mathrm{P} 450$ genes in Culex mosquitoes following treatment with permethrin at their respective $\mathrm{LC}_{50}$ concentrations $(0.005 \mathrm{ppm}$ and $0.7 \mathrm{ppm}$ for the $\mathrm{S}-\mathrm{Lab}$ and $\mathrm{MAmCq}{ }^{\mathrm{G} 6}$ strains, respectively) were analyzed $12,24,48$, and $72 \mathrm{~h}$ after the permethrin treatment. The relative levels of gene expression shown along the $Y$ axis represent the ratio of the gene expression in each treatment in comparison with that in acetone treated control mosquitoes (noting that the $Y$ axis vary among the sub-figures). The experiments were repeated three times. The results are shown as the mean $\pm S$.E. The significant difference was

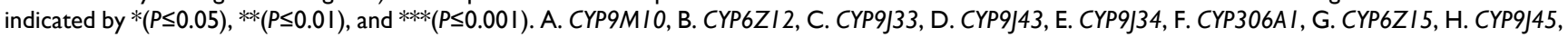
I. CYP9ALI, G. CYP4C52vI, K. CYP9J39. 


\section{Discussion}

Increased levels of P450 gene expression are known to result in increased levels of total P450s and the P450 activities responsible for insecticide resistance $[3,5,6,8,9,34]$. It has been suggested that both constitutively increased expression and induction of P450s in insects are responsible for increased levels of detoxification of insecticides, evolutionary insecticide selection, and the ability of insects to adapt to changing environments $[1,6,33,39,40]$. In a previous study [12], a whole transcriptome analysis of P450 gene expression analysis was used to identify multiple P450 genes that are constitutively overexpressed in insecticide resistance resistant $C x$. quinquefasciatus. Liu et al. [13] further characterized the constitutive overexpression and induction of CYP6AA7, CYP9J34, and CYP9M10 by permethrin in HAmCqG8 Culex mosquitoes, revealing that multiple P450 genes are up-regulated in insecticide resistant mosquitoes through both constitutive overexpression and induction mechanisms, thus increasing the overall expression levels of P450 genes. The current study developed this further by characterizing the induction of P450 genes that were constitutively over-expressed in resistance through a whole genome analysis of resistant $C x$. quinquefasciatus [12]. Two different highly resistant Culex mosquito strains, HAmCqG8 (19 P450 genes) and MAmCqG6 (14 P450 genes), were examined to test our hypothesis that insecticide resistant mosquitoes may be uniquely resistant to insecticides due to their ability of both constitutive overexpression and induction of P450 genes [13], and also allow us to test whether this induction is a common feature for different populations of mosquitoes. We found that seven and eleven P450 genes were significantly induced in the resistant $\mathrm{HAmCq}^{\mathrm{G} 8}$ and MAmCqG6 mosquito strains, respectively, in response to their corresponding $\mathrm{LC}_{50}$ concentrations of permethrin. We also found that three P450 genes, CYP9J34, CYP9M10, and CYP9J45, were induced in both HAmCqG8 and MAmCqG6. These findings are not only consistent with our previous identification of $\mathrm{P} 450$ gene expression in resistant mosquitoes [13] but also support our contention that the induction and constitutive overexpression of these P450 genes plays an important role in the detoxification of insecticides and the development of insecticide resistance as a result of the overall increased expression levels of multiple P450 genes in resistant mosquitoes. We restricted the induction response to permethrin treatment here because this is the insecticide that these mosquitoes are resistant to. P450 gene induction in mosquitoes follows a resistance-specific pattern, where mosquitoes can become resistant to an insecticide through in- creased expression of their P450s as a result of exposure to that insecticide. Similar results have been reported in Drosophila melanogaster, where CYP6g1 and CYP12d1 mRNAs were induced in DDT resistant strains post-exposure to DDT and the expression patterns of these P450 genes varied across DDT resistant strains [59]. Studies by Zhu et al. [9, 35] have also indicated that several P450 genes are up-regulated in insecticide resistant house flies, with a very similar pattern to that observed in studies in mosquitoes. Taken together, these findings strongly suggest a common mechanism for $\mathrm{P} 450$ induction in response to detoxification-mediated insecticide resistance in a number of different insect species.

The results from the current study reveal that different sets of $\mathrm{P} 450$ genes are involved (overexpressed) in different resistant strains (HAmCqG8 and MAmCqG6). This phenomenon has also been reported among different pyrethroid resistant strains of house flies [10]. It has been suggested that the molecular basis of P450-mediated detoxification may be different among different house fly strains $[10,60]$ and resistant mosquito strains (HAmCqG8 and MAmCqG6) may follow the same pattern. This phenomenon has been hypothesized to arise as a result of the evolutionary plasticity of $\mathrm{P} 450$-mediated insecticide resistance $[10,60]$. However, we found that CYP9J45 was induced by permethrin in both the resistant strains of $\mathrm{HAmCq}^{\mathrm{G} 8}$ and MAmCqG6 and CYP9M10, which was found to be induced by permethrin and constitutively overexpressed [13] in resistant HAmCqG8, was also induced significantly in MAmCqG6 in response to permethrin when compared to the susceptible S-Lab strain. Moreover, the overexpression of CYP9M10 has also been reported in a resistant Culex mosquito strain in Japan [61], and has been further linked with pyrethroid resistance in $\mathrm{Cu}$ lex mosquitoes [14, 62]. The coincidence of the induction and constitutive overexpression of CYP9J45 and CYP9M10 in different resistant Culex mosquito strains/populations strongly suggests a common feature of these two P450 genes in pyrethroid resistance in Culex mosquitoes.

It has been suggested that similar regulatory mechanisms may govern P450 constitutive overexpression and induction, and that both contribute to the development of insecticide resistance [27, 33]. Although the mechanisms of regulation for constitutive overexpression and for induction are not clear in insects, it has been reported that in mammals, the constitutive androstane receptor (CAR) and the pregnane $x$ receptor $(\mathrm{PXR})$ have been identified as key transcription factors (IFs) regulating phenobarbital (PB) induced transcription of P450s [25, 63, 64]. A recent study by Lin et al. [65] reported that Drosophila 
hormone receptor-like in 96 (HR96), a single orthologue of CAR and PXR, can act as an activator regulating the $\mathrm{PB}$ induced transcription of cytochrome P450 CYP6D1 in house flies. Abass et al. [66] reported that some P450 genes (CYP3A4, CYP2B6 and CYP2A6) in HepaRG cells could be induced by pyrethroids through both PXR and CAR, and PXP is predominantly activated by pyrethroids rather than other insecticides. Whether a similar mechanism is involved in the induction/overexpression of P450s in mosquitoes is questionable and requires further investigation. It has been suggested that the induction of CYPs is a complex phenomenon involving multiple DNA-binding elements in each P450 promoter, transcriptional factors, and the overlap of regulatory pathways [25]. It will, therefore, be particularly interesting to further characterize transcriptional elements in the promoter region of P450 genes and regulatory factors involved in the regulation of both the constitutive overexpression and induction of the P450 genes implicated in the development of insecticide resistance in order to fully understand the molecular mechanisms involved.

\section{Acknowledgments}

The project described was supported by Award Number R21AI076893 to N.L. from the National Institute of Allergy and Infectious Diseases, AAES Hatch/Multistate Grants ALA08-045 and ALA015-110026 to N.L.

\section{Competing Interests}

The authors have declared that no competing interest exists.

\section{References}

1. Pavek P, Dvorak Z. Xenobiotic-induced transcriptional regulation of xenobiotic metabolizing enzymes of the cytochrome P450 superfamily in human extrahepatic tissues. Curr Drug Metab. 2008; 9: 129-143.

2. Scott JG. Insect Cytochrome P450s: thinking beyond detoxification. In: Liu N Ed. Recent Advances in Insect Physiology, Toxicology and Molecular Biology. Kerala: Research Signpost; 2008: 117-124.

3. Feyereisen R. Insect cytochrome P450. In: Gilbert LI, Iatrou K Eds. Comprehensive Insect Physiology, Biochemistry, Pharmacology and Molecular Biology. Amsterdam: Elsevier; 2005: 1-77.

4. Feyereisen R. Insect CYP genes and P450 enzymes. In: Gilbert LI Ed. Insect Biochemistry Molecular Biology. Elsevier Academic Press; 2012: 236-316.

5. Carino FA, Koener JF, Plapp Jr FW, Feyereisen R. Constitutive overexpression of the cytochrome P450 gene CYP6A1 in a house fly strain with metabolic resistance to insecticides. Insect Biochem Mol Biol. 1994; 24: 411-418.

6. Liu N, Scott JG. Phenobarbital induction of CYP6D1 is due to a trans acting factor on autosome 2 in house flies, Musca domestica. Insect Mol Biol.1997; 6: 77-81.

7. Liu N, Scott JG. Increased transcription of CYP6D1 causes cytochrome P450-mediated insecticide resistance in house fly. Insect Biochem Mol Biol.1998; 28: 531-535.

8. Zhu F, Liu N. Differential expression of CYP6A5 and CYP6A5v2 in pyrethroid-resistant house flies, Musca domestica. Arch Insect Biochem Physiol. 2008; 34: 147-161.
9. Zhu F, Feng JN, Zhang L, Liu N. Characterization of two novel cytochrome P450 genes in insecticide-resistant house-flies. Insect Mol Biol. 2008; 17(1): 27-37.

10. Gao Q, Li M, Sheng C, Scott JG, Qiu X. Multiple cytochrome P450s overexpressed in pyrethroid resistant house flies (Musca domestica). Pestic Biochem Physiol. 2012; 104: 252-260.

11. Kasai S, Weerashinghe IS, Shono T, Yamakawa M. Molecular cloning, nucleotide sequence, and gene expression of a cytochrome P450 (CYP6F1) from the pyrethroid-resistant mosquito, Culex quinquefasciatus Say. Insect Biochem Mol Biol. 2000; 30: 163-171.

12. Yang T, Liu N. Genome analysis of cytochrome P450s and their expression profiles in insecticide resistant mosquitoes, Culex quinquefasciatus. PLoS ONE. 2011; 6(12): e29418.

13. Liu N, Li T, Reid WR, Yang T, Zhang L. Multiple cytochrome P450 Genes: Their constitutive overexpression and permethrin induction in insecticide resistant mosquitoes, Culex quinquefasciatus. PLoS ONE. 2011; 6(8): e23403.

14. Hardstone MC, Komagata O, Kasai S, Tomita T, Scott GJ. Use of isogenic strains indicates CYP9M10 is linked to permethrin resistance in Culex pipiens quinquefasciatus. Insect Mol Biol. 2010; 19: 717-726.

15. Daborn $\mathrm{P}$, et al. A single $\mathrm{P} 450$ allele associated with insecticide resistance in Drosophila. Science. 2002; 297: 2253-2256.

16. Daborn PJ, Lumb C, Boey A, Wong W, ffrench-Constant RH, Batterham P. Evaluating the insecticide resistance potential of eight Drosophila melanogaster cytochrome P450 genes by transgenic over-expression. Insect Biochem Mol Biol. 2007; 37: 512-519.

17. Bariami V, Jones CM, Poupardin R, Vontas J, Ranson H. Gene amplification, $\mathrm{ABC}$ transporters and cytochrome P450s: unraveling the molecular basis of pyrethroid resistance in the dengue vector, Aedes aegypti. PLoS Negl Trop Dis. 2012; 6(6): e1692.

18. Mitchell SN, Stevenson BJ, Müller P, Wilding CS, Egyir-Yawson A, Field SG, Hemingway J, Paine MJ, Ranson H, Donnelly MJ. Identification and validation of a gene causing cross-resistance between insecticide classes in Anopheles gambiae from Ghana. Proc Nat Acad Sci. 2012; 109: 6147-6152

19. Yang Y, Chen S, Wu S, Yue L, Wu Y. Constitutive overexpression of multiple cytochrome $\mathrm{P} 450$ genes associated with pyrethroid resistance in Helicoverpa Armigera. J Econ Entomol. 2006; 99(5): 1784-1789.

20. Zhu F, Parthasarathy R, Bai H, Woithe K, Kaussmann M, Nauen R, Harrison DA, Palli SR. A brain specific cytochrome P450 responsible for the majority of deltamethrin resistance in the QTC279 strain of Tribolium castaneum. Proc Natl Acad Sci USA. 2010; 107: 8557-8562.

21. Karunker I, Benting J, Lueke B, Ponge T, Nauen R, Roditakis E, Vontas J, Gorman K, Denholm I, Morin S. Over-expression of cytochrome P450 CYP6CM1 is associated with high resistance to imidacloprid in the B and $\mathrm{Q}$ biotypes of Bemisia tabaci (Hemiptera: Aleyrodidae). Insect Biochem Mol Biol. 2008; 38: 634-634.

22. Zhuang HM, Wang KF, Zheng L, Wu ZJ, Miyata T, Wu G. Identification and characterization of a cytochrome P450 CYP6CX1 putatively a ssociated with insecticide resistance in Bemisia tabaci. Insect Sci. 2010; 18: 484-494.

23. Karatolos N, Williamson MS, Denholm I, Gorman K, ffrench-Constant $\mathrm{RH}$, et al. Over-expression of a cytochrome P450 is associated with resistance to pyriproxyfen in the greenhouse whitefly Trialeurodes vaporariorum. PLoS ONE. 2012; 7(2): e31077.

24. Reid WR, Zhang L, Liu F, Liu N. The transcriptome profile of the mosquito Culex quinquefasciatus following permethrin selection. PLoS ONE. 2012; 7(10): e47163.

25. Tompkins LM, Wallace AD. Mechanisms of cytochrome P450 induction. J Biochem Mol Toxicol. 2007; 21(4): 176-181.

26. Brattsten LB. Ecological significance of mixed-function oxidations. Drug Metab Rev. 1979; 10: 35-58.

27. Terriere LC. Enzyme induction, gene amplification, and insect resistance to insecticides. In: Geor ghiou GP, Saito T eds. Pest Resistance to Pesticides. New York: Plenum Press; 1983: 265-297

28. Scott JG, Liu N, Wen Z. Insect cytochrome P450: diversity, insecticide resistance and tolerance to plant toxins. Comp Biochem Physiol. 1998; 121: 147-155.

29. Li X, Berenbaum MR, Schuler MA. Cytochrome P450 and actin genes expressed in Helicoverpa zea and Helicoverpa armigera: paralogy/orthology identification, gene conversion and evolution. Insect Biochem Mol Biol. 2002; 32: 311-320.

30. Wen Z, Pan L, Berenbaum MB, Schuler MA. Metabolism of linear and angular furanocoumarins by Papilio polyxenes CYP6B1 co-expressed with NADPH cytochrome P450 reductase. Insect Biochem Mol Biol. 2003; 33: 937-947.

31. David JP, Boyer S, Mesneau A, Ball A, Ranson H, Dauphin-Villemant C. Involvement of cytochrome P450 monooxygenases in the response of mosquito larvae to dietary plant xenobiotics. Insect Biochem Mol Biol. 2006; 36: 410-420. 
32. Schuler MA. Review P450s in plant-insect interactions. Biochim Biophys Acta. 2011; 1814: 36-45.

33. Scharf ME, Parimi S, Meinke LJ, Chandler LD, Siegfried BD. Expression and induction of three family 4 cytochrome P450 (CYP4) genes identified from insecticide-resistant and susceptible western corn rootworms, Diabrotica virgifera virgifera. Insect Mol Biol. 2001; 10(2): 139-146.

34. Festucci-Buselli RA, Carvalho-Dias AS, de Oliveira-Andrade M, Caixeta-Nunes C, Li HM, et al. Expression of Cyp6g1 and Cyp12d1 in DDT resistant and susceptible strains of Drosophila Mela-nogaster. Insect Mol Biol. 2005; 14: 69-77.

35. Zhu F, Li T, Zhang L, Liu N. Co-up-regulation of three P450 genes in response to permethrin exposure in permethrin resistant house flies, Musca domestica. BMC Physiology. 2008; 8(18): 1-13.

36. Bautista M, Tanakaa T, Miyata T. Identification of permethrin-inducible cytochrome P450s from the diamondback moth, Plutella xylostella (L.) and the possibility of involvement in permethrin resistance. Pestic Biochem Physiol. 2007; 87: 85-93.

37. Poupardin R, Reynaud S, Strode C, Ranson H, Vontas J, David JP. Cross-induction of detoxification genes by environmental xenobiotics and insecticides in the mosquito Aedes aegypti: impact on larval tolerance to chemical insecticides. Insect Biochem Mol Biol. 2008; 38: 540 -551.

38. Poupardin R, Riaz MA, Vontas J, David JP, Reynaud S. Transcription profiling of eleven cytochrome $\mathrm{P} 450$ s potentially involved in xenobiotic metabolism in the mosquito Aedes aegypti. Insect Mol Biol. 2010; 19(2): 185-193.

39. Terriere LC. Induction of detoxication enzymes in insects. Ann Rev Entomol. 1984; 29: 71-88.

40. Liu N, Fang Zhu. House Fly Ctyochrome P450s: Their role in insecticide resistance and strategies in the isolation and characterization. In: Liu TX, Kang L Eds. Recent Advances in Entomological Research. Beijing: Higher Education Press and Springer-Verlag GmbH Berlin Heidelberg; 2012: 246-257.

41. Sardelis MR, Turell MJ, Dohm DJ, O'Guinn ML. Vector competence of selected North American Culex and Coquillettidia mosquitoes for West Nile virus. Emerg Infect Dis. 2001; 7: 1018-1022.

42. Jones SC, Morris J, Hill G, Alderman M, Ratard RC. St. Louis encephalitis outbreak in Louisiana in 2001. J La State Med Soc. 2002; 154: 303-306.

43. Reisen WK, Fang Y, Martinez VM. Avian host and mosquito (Diptera: Culicidae) vector competence determine the efficiency of West Nile and St. Louis Encephalitis virus transmission. J Med Entomol. 2005; 42: 367-375.

44. David JP, Ismail HM, Chandor-Proust A, Paine MJI. Role of cytochrome P450s in insecticide resistance: impact on the control of mosquito-borne diseases and use of insecticides on Earth. Phil Trans R Soc B. 2013; 368(1612): 20120429.

45. Najera JA, Zaim M. Malaria vector control: insecticides for indoor residual spraying. WHO document WHO/C DS/WHOP ES/2001; World Health Organization, Geneva.

46. Phillips RS. Current status of malaria and potential for control. Clin Microbiol Rev. 2001; 14: 208-226.

47. Hemingway J, Field L, Vontas J. An overview of insecticide resistance. Science. 2002; 298: 96-97.

48. Liu H, Cupp EW, Micher KM, Guo A, Liu N. Insecticide resistance and cross-resistance in Alabama and Florida strains of Culex quinquefasciatus. J Med Entomol. 2004; 41: 408-413.

49. Xu Q, Liu H, Zhang L, Liu N. Resistance in the mosquito, Culex quinquefasciatus, and possible mechanisms for resistance. Pest Manag Sci. 2005; 61: 1096-1102.

50. Liu N. Insecticide resistance in mosquitoes: development and mechanisms. In: Liu N Ed. Recent Advances in Insect Physiology, Toxicology and Molecular Biology. Research Signpost; 2008: 75-91.

51. Liu N, Xu Q, Li T, He L, Zhang L. Permethrin resistance and target site insensitivity in the mosquito Culex quinquefasciatus in Alabama. J Med Entomol. 2009; 46: 1424-1429.

52. $\mathrm{Xu} \mathrm{Q}$, Wang $\mathrm{H}$, Zhang L, Liu N. Kdr allelic variation in pyrethroid resistance mosquitoes, Culex quinquefasciatus (S). Biochem Biophy Resear Comm. 2006; 345: 774-780.

53. Li T, Liu N. Inheritance of permethrin resistance in Culex quinquefasciatus. J Med Entomol. 2010; 47(6): 1127-1134.

54. Nayar JK, Knight JW. Aedes albopictus (Diptera: Culicidae): an Experimental and Natural Host of Dirofilaria immitis (Filarioidea: Onchocercidae) in Florida, U.S.A. J Med Entomol. 1999; 36(4): 441-448.

55. Wittwer CT, Herrmann MG, Moss AA, Rasmussen RP. Continuous fluorescence monitoring of rapid cycle DNA amplification. BioTechniques. 1997; 22: 130-131.

56. Livak KJ, Schmittgen TD. Analysis of relative gene expression data using real-time quantitative PCR and the $2-\triangle \triangle C T$ method. Methods. 2001; 25: 402-408.
57. Aerts JL, Gonzales MI, Topalian SL. Selection of appropriate control genes to assess expression of tumor antigens using real-time RT-PCR. BioTechniques. 2004; 36: 84-86.

58. Strode C, Wondji CS, David JP, Hawkes NJ, Lumjuan N, Nelson DR, Drane DR, Karunaratne SH, Hemingway J, Black IV WC, Ranson H. Genomic analysis of detoxification genes in the mosquito Aedes aegypti. Insect Biochem Mol Biol. 2008; 38: 113-123.

59. Brandt A, Scharf M, Pedra JHF, Holmes G, Dean A, Kreitman M, Pittendrigh BR. Differential expression and induction of two Drosophila cytochrome P450 genes near the Rst(2) DDT locus. Insect Mol Biol. 2002; 11(4): 337-341

60. Scott JG, Kasai S. Evolutionary plasticity of monooxygenase-mediated resistance. Pestic Biochem Physiol. 2004; 78: 171-178.

61. Komagata O, Kasai S, Tomita T. Overexpression of cytochrome P450 genes in pyrethroid-resistant Culex quinquefasciatus. Insect Biochem Mol Biol. 2010; 40: 146-152.

62. Itokawa K, Komagata O, Kasai S, Okamura Y, Masada M, Tomita T. Genomic structures of Cyp9m10 in pyrethroid resistant and susceptible strains of Culex quinquefasciatus. Insect Biochem Mol Biol. 2010; 40: 631-640.

63. Sueyoshi T, Negishi M. Phenobarbital response elements of cytochrome P450 genes and nuclear receptors. Annu Rev Pharmacol Toxicol. 2001; 41: $123-143$.

64. Timsit YE, Negishi M. CAR and PXR: the xenobiotic-sensing receptors. Steroids. 2007; 72: 231-246.

65. Lin GGH, Kozaki T, Scott JG. Hormone receptor-like in 96 and Broad-Complex modulate phenobarbital induced transcription of cytochrome P450 CYP6D1 in Drosophila S2 cells. Insect Mol Biology. 2011; 20(1): 87-95.

66. Abass K, Lämsä V, Reponen P, Küblbeck J, Honkakoski P, Mattila S, Pelkonen O, Hakkola J. Characterization of human cytochrome P450 induction by pesticides. Toxicology. 2012; 294: 17-26 